\title{
Loss of control eating in adolescents from the community
}

Nora Schlüter, Ricarda Schmidt, Rebekka Kittel, Anne Tetzlaff, Anja Hilbert

This is the peer reviewed version of the following article: Loss of control eating in adolescents from the community, which has been published in final form at http://dx.doi.org/10.1002/eat.22488. This article may be used for non-commercial purposes in accordance with Wiley Terms and Conditions for Self-Archiving. [Link to: http://olabout.wiley.com/WileyCDA/Section/id-820227.html\#terms]

Integrated Research and Treatment Center AdiposityDiseases, Department of Medical

Psychology and Medical Sociology, Leipzig University Medical Center

LOC eating in adolescents

Abstract word count: 198

Word count: 4044

\section{Correspondence:}

Anja Hilbert, PhD, Leipzig University Medical Center Integrated Research and

Treatment Center AdiposityDiseases, Department of Medical Psychology and Medical

Sociology, Philipp-Rosenthal-Strasse 27, 04103 Leipzig, Germany

Telephone: +49 34197 15360, Fax: +493419715359

E-mail: Anja.Hilbert@medizin.uni-leipzig.de 


\begin{abstract}
Objective: Loss of control (LOC) eating is a salient indicator of eating disorder psychopathology in adolescents and is associated with marked distress. While research has focused on the relevance of episode size, clinical significance of LOC eating frequency has rarely been explored. Therefore, this study aimed at identifying LOC eating prevalence with respect to its recurrence and associated variables in a community-based sample.

Method: Participants were 1643 adolescents, aged 12-20 years (62.4\% female). Based on EDE-Q self-report, participants were categorized as those reporting recurrent $(N=156$; 9.5\%), non-recurrent $(N=226 ; 13.8 \%)$ and no LOC eating $(N=1261 ; 76.7 \%)$.

Results: Adolescents with recurrent LOC eating reported clinically relevant and significantly greater eating disorder psychopathology, functional impairment and distress because of LOC eating, and a significantly higher body mass index $\left(\mathrm{BMI}, \mathrm{kg} / \mathrm{m}^{2}\right)$ than adolescents with nonrecurrent and those without LOC eating.

Discussion: These results underline that LOC eating is a common eating behavior among adolescents in the community associated with clinical characteristics of eating disorders, and could therefore be a risk factor for developing full- or partial-syndrome eating disorders. Further research on the classification of eating disorders in adolescents with LOC eating and severity indicators is warranted.
\end{abstract}

Keywords: loss of control eating; adolescents; obesity; binge-eating disorder; screening 


\section{LOSS OF CONTROL EATING IN ADOLESCENTS FROM THE COMMUNITY}

Previous research indicates that loss of control (LOC) eating, defined as the experience of being unable to control one's eating irrespective of the amount of food consumed, is a common disturbed eating behavior in adolescents ${ }^{1-5}$ that is associated with elevated eating disorder psychopathology and distress. ${ }^{1-3,6}$ However, research is needed to more fully describe demographic characteristics and clinical features of adolescents who report LOC eating, especially regarding varying levels of LOC eating frequency in the community. Therefore, the current study aimed to provide data on prevalence, epidemiological and clinical associations of LOC eating in an adolescent community sample.

Binge eating is a core feature of several eating disorders that is characterized by the consumption of a large amount of food associated with feelings of LOC over eating. ${ }^{7}$ Although the Diagnostic and Statistical Manual of Mental Disorders, fifth edition (DSM-5), ${ }^{7}$ still declares binge eating the defining criterion of binge-eating episodes, the upcoming eleventh revision of the International Classification of Diseases (ICD-11) proposes that LOC eating is the core defining feature of both subjective and objective binge-eating episodes. ${ }^{8}$ Converging evidence suggests that LOC eating, rather than episode size, to be the most salient indicator of eating disorder psychopathology associated with marked distress in adolescent community and overweight patient samples. ${ }^{1-3,6}$

Although adolescence has been shown to be a vulnerable developmental stage for the onset of eating disorders, ${ }^{9,10}$ only few studies have examined additional epidemiological correlates of LOC eating. Interview-based studies have found that adolescent LOC eating is highly prevalent in overweight boys and girls (ranging from 30\% to 39\%). ${ }^{2,4,5}$ Lower prevalence estimates have been reported in community samples across a range of ages and not specifically selected based on overweight status; ${ }^{1,3}$ however, among certain subgroups prevalence of LOC eating was still considerable, for example among girls or individuals with 
low socio-economic status. ${ }^{1,3}$ While diagnosing an eating disorder requires clinical interview assessment such as the Eating Disorder Examination (EDE), ${ }^{11}$ self-report questionnaires represent an economical and non-intrusive approach to assess eating disorder psychopathology, especially within epidemiological frameworks. One well-established selfreport measure on specific eating disorder psychopathology and diagnostically relevant behavioral features including loss of control and binge eating is the Eating Disorder Examination-Questionnaire (EDE-Q) $)^{3,12}$ that was derived from the EDE.

Current research aimed at identifying severity criteria for LOC eating in adolescence, similar to the DSM-5 frequency threshold of binge-eating episodes in adult binge-eating disorder (BED). ${ }^{13-16}$ In adolescent samples, previous studies primarily focused on episode size, ${ }^{5,6,17,18}$ while the clinical significance of LOC eating frequency has rarely systematically been explored in the population. ${ }^{3,19}$ First evidence from adolescent overweight patient samples $^{2,4}$ and a child community sample ${ }^{19}$ indicated that those who reported recurrent versus non-recurrent LOC eating showed more eating disorder and general psychopathology. A study on adolescents from the community, however, did not find these associations. ${ }^{3}$ Thus, elucidating the role of LOC eating frequency on psychopathology might help to clarify whether this criterion might be a useful indicator of LOC eating severity in youth and to identify those in need for early intervention.

In this context, this study aimed to provide prevalence data on three frequency levels of adolescent LOC eating (no, non-recurrent, and recurrent LOC eating) and to investigate demographic and clinical correlates of these levels of LOC eating in adolescents. We hypothesized that adolescents with LOC eating, especially those with recurrent LOC eating, were more likely to be female, to report lower levels of education, a higher BMI, and higher levels of eating disorder psychopathology and impairment due to eating disorders psychopathology relative to adolescents without LOC eating. 


\section{METHOD}

\section{$\underline{\text { Participants and Procedure }}$}

Participants aged 12-20 years were recruited from the community through local schools from March 2013 to July 2014. The German educational system places adolescents into low, intermediate, and high academic tracks, so that the students' proficiency levels differ between the different types of schools (Hauptschule, Realschule, and Gymnasium). In the present study, all 55 schools in Leipzig, Saxony, were contacted. Out of these, 18 schools agreed to participate (total school response rate 32.7\%), including ten schools from the lower and intermediate secondary tracks (Haupt-/Realschule; track-specific response rate 31.3\%) and eight schools from the higher secondary track (Gymnasium; track-specific response rate 34.8\%). For the classroom-based assessment, classes were chosen according to availability. The better response of higher secondary track schools resulted in the participation of 703 adolescents from higher secondary track and 492 from lower and intermediate secondary tracks. In order to capture LOC eating in older adolescents (18 to 20 years), adolescents from nine vocational schools (track-specific response rate 13.0\%) and a student sample were recruited. Three medical semesters ( $n=898$ medical students) of the University of Leipzig were contacted, of whom 302 students agreed to participate (response rate: 33.6\%). Prior to participation, all families received detailed information on the study aims and procedures; assent of adolescents $<18$ years and written informed consent from adolescents $\geq 18$ years and of one parent for adolescents $<18$ years was obtained. During classes, training courses, and university courses, the questionnaire was administered by doctoral students to adolescents who completed the questionnaire by themselves and anonymously. Ethical approval of this study was granted through the ethics committee of the Medical Faculty of the University of Leipzig, Germany. 


\section{Classificatory Variables}

LOC eating. For identifying LOC eating episodes, the 2-part question of the Eating Disorder Examination-Questionnaire (EDE-Q), a well-established self-report questionnaire for assessing specific psychopathology and key behavioral features of eating disorders, ${ }^{12,20}$ was administered ("How many times have you eaten what other people regard as an unusually large amount of food (given the circumstances)?”, and “On how many of these times did you have a sense of having lost control over your eating (at the time that you were eating)?”). Self-report of binge eating in youth was found less valid compared to interview-based assessment leading to an overestimation of BED in youth ${ }^{3}$ compared to interview-based assessment. ${ }^{10,21}$ Relatedly, determining an “unambiguously large amount of food” for BED diagnosis in youth can be difficult ${ }^{18}$ due to varying nutritional needs in growth and limited accessibility of food. LOC eating seems therefore to be a more salient feature of adolescent binge eating including those with both subjective and/or objective binge-eating episodes. Therefore, the present study primarily focused on LOC eating rather than binge eating in youth.

For classificatory analysis, three groups were determined based on the frequency of LOC eating episodes during the last 28 days. The first group included adolescents without any LOC eating episode (NoLOC). Following the DSM-5 frequency criterion of binge-eating episodes in BED (“at least once a week”) ${ }^{7}$ and recent classificatory proposals on objective and subjective binge-eating episodes in young adults ${ }^{22-24}$, the second group included adolescents with at least 4 LOC eating episodes during the last month ( $\geq 4$ episodes; recurrent LOC). Furthermore, according to the specific presentation "BED of low frequency and/or limited duration" characterized by "binge eating less than once a week" (DSM-5), ${ }^{7}$ the third group included adolescents with non-recurrent LOC eating (1 to 3 episodes; non-recurrent LOC). 
Binge-eating disorder. For exploratory purposes, it was analyzed how many adolescents with LOC eating would meet criteria for BED (according to DSM-5). ${ }^{7}$ For identifying binge-eating episodes, the following item of the EDE-Q was used "On how many DAYS have such episodes of overeating occurred (i.e., you have eaten an unusually large amount of food and have had a sense of loss of control at the time)?”. According to the diagnostic criteria of BED, seven items from the Eating Disorder Examination (EDE) were applied to measure behavioral characteristics of binge eating (eating more rapidly, eating alone, eating when not hungry, eating until uncomfortably full, and feeling guilty and depressed after eating), distress due to binge eating, and compensatory behaviors over the last 28 days, rated on a dichotomous scale with present $=0$, absent $=1$. Full-syndrome BED was defined as at least 4 binge-eating episodes during the last 28 days, $\geq 3$ behavioral characteristics and distress because of BED, while partial-syndrome BED was defined as at least 1 binge-eating episode during the last 28 days and $<3$ behavioral characteristic or lack of distress because of BED, without claiming to derive a BED diagnosis comparable to interview assessment.

\section{$\underline{\text { Eating Disorder Psychopathology }}$}

Eating Disorder Examination-Questionnaire 8 (EDE-Q8). For an economical assessment of global eating disorder psychopathology the short version of the EDE-Q (EDEQ8) was used, ${ }^{25}$ a brief validated version of the EDE-Q containing 8 of the original 28 items of the EDE-Q (2 items of each subscale Restraint, Eating Concern, Shape Concern, and Weight Concern) rated on a seven-point Likert scale $(0=$ not at all to $6=$ every day $)$ and referring to the last 28 days. A mean global score was calculated, ranging from 0 to 6 , with higher scores indicating greater psychopathology. Based on a cutoff of 3.13 ( $90^{\text {th }}$ percentile), the EDE-Q8 discriminates between individuals at high vs. low risk for clinically significant 
eating disorder symptoms. ${ }^{25}$ In the present study, internal consistency was good (Cronbach's $\alpha=0.89)$.

Clinical Impairment Assessment (CIA). The CIA is a 16-item self-report measure to assess the severity of psychosocial impairment due to eating disorder psychopathology on three subscales (Personal, Social, and Cognitive Impairment). ${ }^{26}$ All items refer to the last 28 days, and were rated on a 4-point Likert scale ranging from $0=$ not at all to $3=a$ lot. A global sum score was calculated ranging from 0 to 48 with higher sum scores indicating a higher level of impairment. In adolescent and adult samples a cutoff score of 16 was found to be a valid indicator of clinical impairment due to an eating disorder. ${ }^{26-29}$ In the present study internal consistency was acceptable (Cronbach's $\alpha=0.72$ ).

\section{Data Analytic Plan}

Analyses were conducted using SPSS Version 20.0. All analyses were performed for group comparisons between adolescents with recurrent LOC, non-recurrent LOC, and no LOC. In order to compare the groups on sex, education and weight status, $\chi^{2}$ tests and, in case of significance, pairwise post-hoc tests were performed. Similarly, univariate analyses of variance (ANOVA) and subsequent independent $t$ tests in case of significance were performed on age and BMI-SDS. Hypothesized group differences on eating disorder psychopathology and impairment (EDE-Q8, CIA) were analyzed using multivariate analyses of covariance (MANCOVA) with corrections for sociodemographic (sex, education) and anthropometric (BMI-SDS) variables on which the groups differed significantly (see below). For post-hoc comparisons, Tukey HSD tests were used. Differences on behavioral symptoms of LOC eating between the recurrent and non-recurrent LOC eating groups were computed using $\chi^{2}$ tests. Effect sizes were interpreted as small $\left(0.01 \leq \eta^{2}<0.06\right.$; Cramer's V $\left.=0.10\right)$, medium $\left(0.06 \leq \eta^{2}<0.14\right.$; Cramer's $\left.V=0.30\right)$, and large $\left(\eta^{2} \geq 0.14\right.$; Cramer's $\left.V=0.50\right)$ according to 
Cohen (1988). A two-tailed $\alpha<.05$ was applied for significance. For exploratory purposes, adolescents with any LOC eating were compared on meeting full- or partial-syndrome BED criteria using $\chi^{2}$ tests.

\section{RESULTS}

A total of 3362 students were eligible for study participation, of which 1643 (48.9\%) students consented to participate. The total study sample (female: $n=1025,62.4 \%$ ) had a mean age of 15.0 years $(S D=2.8)$. The mean BMI of $20.4 \mathrm{~kg} / \mathrm{m}^{2}(S D=3.3)$, and the mean BMI-Standard Deviation Score (BMI-SDS) of -0.07 $(S D=0.9)$ were calculated based on selfreported height and weight ( $n=53,3.2 \%$ incomplete anthropometric data). According to German age- and sex-related reference data ${ }^{30} 8.5 \%(n=135)$ of the adolescents were classified as being underweight (below the $10^{\text {th }}$ percentile), the majority of $81.3 \%(n=1292)$ as being normal weight (between the $10^{\text {th }}$ and $90^{\text {th }}$ percentile), $7.7 \%(n=122)$ as being overweight (above the $90^{\text {th }}$ percentile), and $2.6 \%(n=41)$ as being obese (above the $97^{\text {th }}$ percentile). A total of 29.9\% $(n=492)$ attended schools from the lower and intermediate secondary tracks, 42.8\% $(n=703)$ attended schools from higher secondary track, $8.9 \%(n=$ 146) vocational schools and $18.4 \%(n=302)$ university (Table 1$)$.

\section{Prevalence of LOC Eating}

Based on adolescents’ self-report (EDE-Q), 76.7\% $(N=1261)$ reported no LOC eating and 23.3\% $(N=382)$ reported any LOC eating over the last 28 days. Regarding LOC eating frequency, 9.5\% $(N=156)$ reported at least 4 LOC eating episodes (recurrent LOC group), and $13.8 \%(N=226)$ reported at least 1 , but fewer than 4 episodes (non-recurrent LOC group). While the recurrent LOC group reported a mean of 10.99 LOC eating episodes ( $S D=$ 
12.26), the non-recurrent LOC group reported 1.71 LOC eating episodes $(S D=0.74)$ during the past 28 days.

Exploratory analyses revealed that, based on the total sample, 5.1\% $(n=83)$ of adolescents met criteria for full-syndrome BED and 16.8\% $(n=272)$ met criteria for partialsyndrome BED. Regarding LOC subgroups, 41.3\% $(n=62)$ of the recurrent LOC group and $10.2 \%(n=21)$ of the non-recurrent LOC group met criteria for full-syndrome BED, while partial-syndrome BED was identified in $58.7 \%(n=88)$ of the recurrent LOC and in $89.8 \%$ ( $n$ $=184$ ) of the non-recurrent LOC group.

-- Please insert Table 1 here --

\section{$\underline{\text { Sociodemographic Correlates of LOC Eating }}$}

As shown in Table 1, adolescents in the recurrent LOC and non-recurrent LOC group did not differ in sex $(p>.05)$, but both groups showed significantly higher prevalence of girls than the NoLOC group $(p<.001)$. The BMI-SDS was greater in the recurrent LOC group compared to the non-recurrent LOC $(p<.01)$, and NoLOC group $(p<.001)$, while the nonrecurrent LOC group showed a significantly higher BMI-SDS than the NoLOC group $(p<$ .001). Regarding education, adolescents in the recurrent LOC group attended significantly more often schools from lower and intermediate secondary tracks than the non-recurrent LOC $(p<.05)$ and the NoLOC group $(p<.001)$, while the non-recurrent LOC group revealed significantly lower levels of education than the NoLOC group $(p<.01)$. The groups did not differ in age $(p>.05)$. 


\section{$\underline{\text { Clinical Correlates of LOC Eating }}$}

Multivariate analysis showed significant group differences on the EDE-Q8 and CIA, when adjusted for covariates $(F(6,3166)=103.577, p<.001$; Table 2$)$. Regarding post-hoc comparisons, adolescents in the recurrent LOC group scored significantly higher on the EDE-

Q8 and CIA than adolescents in the non-recurrent LOC and NoLOC group (both $p<.001$ ). The non-recurrent LOC group, in turn, showed significantly higher scores on the EDE-Q8 and CIA compared to the NoLOC group $(p<.001)$.

On the categorical level, significantly more adolescents in the recurrent LOC group exceeded cutoff scores on the EDE-Q8 and on the CIA compared to adolescents in the nonrecurrent LOC $(p<.001)$ and NoLOC group $(p<.001)$. Adolescents in the non-recurrent LOC group, in turn, scored significantly more often above the EDE-Q8 and CIA cutoff scores than those in the NoLOC group (all $p<.001$ ).

Regarding behavioral characteristics and distress because of LOC eating, only the recurrent LOC and non-recurrent LOC group were compared. The recurrent LOC group showed significantly more behavioral symptoms of LOC eating than the non-recurrent LOC group ( $p$.001), and reported significantly higher levels of distress because of LOC eating relative to the non-recurrent LOC group $(p<.001)$, while the majority of adolescents with non-recurrent LOC eating reported no distress.

\section{DISCUSSION}

This study provided first epidemiological data of LOC eating in a large community sample of German adolescents. Based on self-reports, 23.3\% of the 12- to 20-year old adolescents from the community reported LOC eating during the last 28 days indicating that LOC eating is a common disordered eating behavior among adolescents. The frequency of LOC eating episodes was found to play an important role regarding LOC eating's clinical 
significance with adolescents endorsing at least 4 LOC eating episodes during the last month to report significantly more eating disorder psychopathology, related distress and a higher BMI-SDS than adolescents with fewer than 4 LOC eating episodes.

Our results are consistent with previous evidence indicating that LOC eating is not only highly prevalent in clinical overweight adolescent samples, ${ }^{2,4,5,31}$ but also in adolescents from the community. ${ }^{1,3}$ The prevalence of LOC eating in the present study (23.3\%) was comparable to a previous community-based study among American adolescents (28.4\%) ${ }^{1}$ using an interview-based assessment. However, it was somewhat higher compared to the only other community-based study using self-report questionnaires $(16.7 \%) .{ }^{3}$ We suggest that the difference in prevalence is related to differences in the samples with adolescents in the Goossens’ study being younger and having higher socioeconomic background than adolescents in the present study.

Prospective findings suggested that LOC eating, rather than the amount of food consumed, predicted the onset of overweight, obesity, and depressive symptoms. ${ }^{32}$ In line with these findings and with cross-sectional evidence, ${ }^{2,4}$ we found recurrent LOC eating to be associated with more adverse health outcomes (i.e., eating disorder psychopathology, higher $\mathrm{BMI}$ ). The clinical significance of the frequency of LOC eating has only been examined in two studies in youth, ${ }^{3,19}$ showing inconsistent findings. Hilbert and Czaja ${ }^{19}$ documented that the classification of high ( $\geq 2$ episodes per month) versus low ( $<2$ episodes per month) recurrent LOC eating in pre-adolescent children, determined based on expert interview, proved to be externally valid, because children with at least 2 LOC eating episodes during the past 28 days already revealed greater eating disorder psychopathology and depression than those with low recurrent LOC eating. Contrary to our results, Goossens et al. ${ }^{3}$ found, based on EDE-Q self-report, that youth from the community with most frequent $(\geq 8$ episodes per month), high frequent (4-7 episodes per month), and low frequent (1-3 episodes per month) 
LOC eating did not differ on eating disorder and general psychopathology. Importantly, the present study was based on a larger sample than these previous studies ${ }^{3,19}$ with greater diversity regarding age and educational level, and approaching representativeness.

Although LOC eating is neither a full-syndrome nor a partial eating disorder as defined in DSM-5, ${ }^{7}$ we found that it is associated with clinically relevant eating disorder psychopathology in youth, particularly for adolescents with recurrent LOC eating episodes. A total of $68.8 \%$ of adolescents with recurrent LOC eating reported an above threshold score for eating disorder psychopathology (EDE-Q8). This rate of clinical significant global eating disorder psychopathology exceeded rates of adolescent and young adult samples from the general population ${ }^{23,24,33}$ and was comparable to findings from clinical adult samples with $\mathrm{BED}^{34}$ or various eating disorders. ${ }^{35}$ The clinical impairment of adolescents with LOC eating, based on the CIA, was within the range of clinical and general population samples. ${ }^{27,28,35}$ As clinical impairment often leads individuals with eating disorders to seek treatment, ${ }^{36}$ higher clinical impairment will probably be found in treatment-seeking samples with LOC eating.

While adolescents in the non-recurrent LOC group reported less behavioral symptoms and the majority of them showed no or little distress because of LOC eating, those with recurrent LOC eating reported approximately three behavioral symptoms and moderate to high distress because of LOC eating symptomatology, suggesting that some of them would nearly or fully meet BED criteria. Indeed, exploratory analyses revealed that about $41 \%$ of adolescents with at least 4 LOC eating episodes during the last month would meet DSM-5 criteria for full-syndrome BED diagnosis, while the remaining 59\% would meet DSM-5 criteria for partial-syndrome BED diagnosis. Adolescents with fewer than 4 LOC eating episodes per month were less likely to meet full-syndrome BED criteria (10\%), but would fulfil DSM-5 criteria for partial-syndrome BED (90\%). Although based on self-reports, which are likely to overestimate prevalence, the results illustrate the importance of LOC eating 
assessment considering LOC eating to predict the onset of full- or partial-syndrome eating disorders. ${ }^{5,18,37,38}$

Beyond prospective evidence suggesting LOC eating to increase the risk for excessive weight gain, ${ }^{38}$ cross-sectional adolescent community-based studies already found positive associations between LOC eating and weight status in youth. ${ }^{1,6}$ Our results are in line with these interview-based studies that found positive associations between BMI-SDS and LOC eating, while demonstrating that adolescents with recurrent LOC eating were even more likely to have elevated BMI-SDS scores compared to those with non-recurrent LOC eating.

The fact that significantly more girls than boys reported LOC eating is consistent with community-based studies. ${ }^{1,3}$ In contrast to one previous prospective finding showing the frequency of LOC eating episodes to increase with age in a clinical sample, ${ }^{18}$ our results are in line with the majority of community-based and treatment-seeking overweight adolescent studies that found no age dependence of LOC eating. ${ }^{1-4}$ The result that a significantly larger proportion of adolescents with LOC eating attended schools from lower and intermediate secondary tracks than schools from the higher secondary track, vocational schools, or university supports the idea that a lower socioeconomic status may represent a general risk factor for psychosocial problems including eating disorders, ${ }^{39}$ which would require longitudinal confirmation. Alternatively, Allen et al. ${ }^{40}$ documented in their longitudinal population-based study that youth with a persistent eating disorder were less likely to complete high school than other students. With regard to its adverse health-related associations (i.e., eating disorder psychopathology, increased BMI-SDS) the results underline the importance of an early screening of LOC eating, similar to findings on other subthreshold eating disorders. ${ }^{41,42}$ At the same time, establishing early intervention for adolescents with LOC eating or BED seems challenging, since binge eating has received little attention from primary health care providers. ${ }^{43}$ 
Strengths of the present study include a large school-based sample of both girls and boys from diverse educational settings across a broad age range (12-20 years old). Thus, our findings extended data from previous studies, which have mostly concentrated on overweight treatment-seeking individuals. Given the sparse evidence of the clinical significance of recurrent and non-recurrent LOC eating, our study was the first that specifically examined LOC subsamples according to episode frequency in a large community-based adolescent sample. Furthermore, standardized self-report questionnaires were used to assess the eating disorder psychopathology and clinical impairment. Behavioral characteristics and distress because of binge eating were assessed with EDE-based self-report, according to the diagnostic criteria for BED (DSM-5). ${ }^{7}$

At the same time, the use of a self-report questionnaire to assess LOC eating limits the interpretation of the study's results. Clinical interviews, such as the EDE, ${ }^{11}$ represent a more valid, but for epidemiological purposes less economical, measure of eating disorder psychopathology. Importantly, school response rates in the present study were relatively low and varied between different types of schools resulting in a higher number of participating students from the higher vs. lower secondary track. Out of the whole student population in Saxony, only $41 \%$ students attend higher secondary track schools, ${ }^{44}$ thus, in this study, students from higher secondary track schools were overrepresented. Although all analyses were adjusted for adolescents’ education, the results were possibly affected by this selection bias and may not be fully generalizable to all adolescents from the community. Due to the relatively low percentage of adolescents from the lower and intermediate secondary track, the present results may underestimate actual LOC eating prevalence in the German community. The prevalence of overweight (7.7\%) and obese (2.3\%) adolescents among this sample was somewhat lower than objectively measured prevalence of German adolescents ${ }^{45}$ and supports evidence that self-reported height and weight lead to an underestimation of adolescents’ 
BMI. ${ }^{46}$ Alternatively, the overrepresentation of youth with higher educational level may have contributed to lower prevalence of overweight and obesity. ${ }^{47}$ Finally, because an interviewbased confirmation of BED diagnosis was lacking, it remains unclear how many adolescents with LOC eating actually meet full- or partial-syndrome criteria for a DSM-5 BED.

In summary, the present study revealed that almost a quarter of all adolescents from the community experienced LOC eating episodes. Adolescents with LOC eating require further attention, because they already showed clinical levels of eating disorder psychopathology, functional impairment, and elevated BMI-SDS, and may therefore be at risk for developing full- or partial-syndrome eating disorders. Longitudinal studies of childhood samples found that LOC eating has moderate persistence, ${ }^{37,48}$ but less is known about the course of adolescent LOC eating. Although the present study provided support for the recently developed BED severity specifier that is based on binge-eating frequency and newly included in the DSM-5, ${ }^{7}$ current evidence highlights the investigation of alternative specifier for BED severity, such as the over-evaluation of shape and weight. ${ }^{13,14}$ Nevertheless, our results support that focusing on the frequency of LOC eating episodes might be useful to identify adolescents with higher severity levels and potentially in need of early intervention. ${ }^{36}$ 
Acknowledgement:

This work was supported by the Federal Ministry of Education and Research (BMBF), Germany, FKZ: 01EO1001.

Conflict of Interest:

No author of this manuscript does have any conflict of interest, financial or otherwise, related to the submitted work. 


\section{References}

1. Elliott CA, Tanofsky-Kraff M, Shomaker LB, Columbo KM, Wolkoff LE, Ranzenhofer LM, et al. An examination of the interpersonal model of loss of control eating in children and adolescents. Behav Res Ther 2010;48,424-428.

2. Goldschmidt AB, Jones M, Manwaring JL, Luce KH, Osborne MI, Cunning D, et al. The clinical significance of loss of control over eating in overweight adolescents. Int J Eat Disord 2008;41:153-158.

3. Goossens L, Soenens B, Braet C. Prevalence and Characteristics of Binge Eating in an Adolescent Community Sample. J Clin Child Adolesc Psychol 2009;38:342-353.

4. Glasofer DR, Tanofsky-Kraff M, Eddy KT, Yanovski SZ, Theim KR, Mirch MC, et al. Binge Eating in Overweight Treatment-Seeking Adolescents. J Pediatr Psychol 2007;32:95-105.

5. Tanofsky-Kraff M, Goossens L, Eddy KT, Ringham R, Goldschmidt A, Yanovski SZ, et al. A multisite investigation of binge eating behaviors in children and adolescents. $\mathrm{J}$ Consult Clin Psychol 2007;75:901-913.

6. Shomaker LB, Tanofsky-Kraff M, Elliott C, Wolkoff LE, Columbo KM, Ranzenhofer LM, et al. Salience of loss of control for pediatric binge episodes: Does size really matter? Int J Eat Disord 2010;43:707-716.

7. American Psychiatric Association. Diagnostic and statistical manual of mental disorders, text revision (5th ed.). Washington, DC: American Psychiatric Association, 2013.

8. Uher R, Rutter M. Classification of feeding and eating disorders: review of evidence and proposals for ICD-11. World Psychiatry 2012;11:80-92. 
9. Stice E, Marti CN, Rohde P. Prevalence, incidence, impairment, and course of the proposed DSM-5 eating disorder diagnoses in an 8-year prospective community study of young women. J Abnorm Psychol 2013;122:445-457.

10. Swanson SA, Crow SJ, Le Grange D, Swendsen J, Merikangas KR. Prevalence and Correlates of Eating Disorders in Adolescents: Results From the National Comorbidity Survey Replication Adolescent Supplement. Arch Gen Psychiatry 2011;68:714-723.

11. Fairburn CG, Cooper Z. The eating disorders examination (12th ed). In: Fairburn CG, Wilson GT, editors. Binge eating: nature, assessment, and treatment. New York: The Guilford Press, 1993, p. 317-331.

12. Fairburn CG, Beglin SJ. The Assessment of Eating Disorders: Interview versus Questionnaire. Int J Eat Disord 1994;16:363-370.

13. Grilo CM, Ivezaj V, White MA. Evaluation of the DSM-5 severity indicator for binge eating disorder in a clinical sample. Beh Res Ther 2015a;71:110-114.

14. Grilo CM, Ivezaj V, White MA. Evaluation of the DSM-5 severity indicator for binge eating disorder in a community sample. Behav Res Ther 2015b;66:72-76.

15. Striegel-Moore RH, Dohm F-A, Solomon EE, Fairburn CG, Pike KM, Wilfley DE. Subthreshold Binge Eating Disorder. Int J Eat Disord 2000;27:270-278.

16. Trace SE, Thornton LM, Root TL, Mazzeo SE, Lichtenstein P, Pedersen NL, et al. Effects of Reducing the Frequency and Duration Criteria for Binge Eating on Lifetime Prevalence of Bulimia Nervosa and Binge Eating Disorder: Implications for DSM-5. Int J Eat Disord 2012;45:531-536. 
17. Goossens L, Braet C, Bosmans G, Decaluwé V. Loss of control over eating in preadolescent youth: The role of attachment and self-esteem. Eat Behav 2011;12:289295.

18. Tanofsky-Kraff M, Shomaker LB, Olsen C, Roza CA, Wolkoff LE, Columbo KM, et al. A prospective study of pediatric loss of control eating and psychological outcomes. J Abnorm Psychol 2011;120:108-118.

19. Hilbert A, Czaja J. Binge eating in primary school children: Towards a definition of clinical significance. Int J Eat Disord 2009;42:235-243.

20. Hilbert A, Tuschen-Caffier B. Eating Disorder Examination-Questionnaire: Deutschsprachige Übersetzung (Bd. 02). Münster: Verlag für Psychotherapie, 2006.

21. Stice E, Marti CN, Shaw H, Jaconis M. An 8-year longitudinal study of the natural history of threshold, subthreshold, and partial eating disorders from a community sample of adolescents. J Abnorm Psychol 2009;118:587-597.

22. Hilbert A, de Zwaan M, Braehler E. How Frequent Are Eating Disturbances in the Population? Norms of the Eating Disorder Examination-Questionnaire. PLoS ONE 2012;7:1-7.

23. Luce KH, Crowther JH, Pole M. Eating Disorder Examination Questionnaire (EDEQ): Norms for undergraduate women. Int J Eat Disord 2008;41:273-276.

24. Mond J, Hay P, Rodgers B, Owen C. Eating Disorder Examination Questionnaire (EDE-Q): Norms for young adult women. Behav Res Ther 2006;44:53-62.

25. Kliem S, Mößle T, Zenger M, Strauss B, Brähler E, Hilber A. The Eating Disorder Examination-Questionnaire 8: A Brief Measure of Eating Disorder Psychopathology (EDE-Q8). Int J Eat Disord (in press). 
26. Bohn K, Fairburn CG. Clinical impairment questionnaire (CIA 3.0). In: Fairburn CG, editor. Cognitive behavior therapy and eating disorders. New York: Guilford Press, 2008, pp. 315-317.

27. Becker AE, Thomas JJ, Bainivualiku A, Richards L, Navara K, Roberts AL, et al. Adaptation and Evaluation of the Clinical Impairment Assessment to Assess Disordered Eating Related Distress in an Adolescent Female Ethnic Fijian Population. Int J Eat Disord 2010;43:179-186.

28. Reas DL, Rø Ø, Kapstad H, Lask B. Psychometric Properties of the Clinical Impairment Assessment: Norms for Young Adult Women. Int J Eat Disord 2010;43:72-76.

29. Vannucci A, Kass AE, Sinton MM, Aspen V, Weisman H, Bailey JO, et al. An examination of the Clinical Impairment Assessment among women at high risk for eating disorder onset. Behav Res Ther 2012;50:407-414.

30. Kromeyer-Hauschild K, Wabitsch M, Kunze D, Geller F, Geiß HC, Hesse V, et al. Perzentile für den Body-mass-Index für das Kindes- und Jugendalter unter Heranziehung verschiedener deutscher Stichproben. Monatsschr Kinderheilkd 2001;807-818.

31. Neumark-Sztainer D, Story M, Resnick MD, Blum RW. Psychosocial Concerns and Weight Control Behaviors Among Overweight and Nonoverweight Native American Adolescents. J Am Diet Assoc 1997;97:598-604.

32. Sonneville KR, Horton NJ, Micali N, Crosby RD, Swanson SA, Solmi F, et al. Longitudinal Associations Between Binge Eating and Overeating and Adverse Outcomes Among Adolescents and Young Adults. JAMA Pediatr 2013;167:149-155. 
33. Carter JC, Stewart DA, Fairburn CG. Eating disorder examination questionnaire: norms for young adolescent girls. Behav Res Ther 2001;39:625-632.

34. Reas DL, Grilo CM, Masheb RM. Reliability of the Eating Disorder ExaminationQuestionnaire in patients with binge eating disorder. Behav Res Ther 2006;44:43-51.

35. Welch E, Birgegård A, Parling T, Ghaderi A. Eating disorder examination questionnaire and clinical impairment assessment questionnaire: General population and clinical norms for young adult women in Sweden. Behav Res Ther 2011;49:85-91.

36. Vannucci A, Theim KR, Kass AE, Trockel M, Genkin B, Rizk M, et al. What constitutes clinically significant binge eating? Association between binge features and clinical validators in college-age women. Int J Eat Disord 2013;46:226-232.

37. Hilbert A, Brauhardt A. Childhood loss of control eating over five-year follow-up. Int J Eat Disord 2014;47:758-761.

38. Stice E, Presnell K, Spangler D. Risk factors for binge eating onset in adolescent girls: A 2-year prospective investigation. Health Psychol 2002;21:131-138.

39. Hölling H, Schlack R. Essstörungen im Kindes- und Jugendalter: Erste Ergebnisse aus dem Kinder- und Jugendgesundheitssurvey (KiGGS). Bundesgesundheitsblatt Gesundheitsforschung Gesundheitsschutz 2007;50:794-799.

40. Allen KL, Byrne SM, Oddy WH, Crosby RD. Early Onset Binge Eating and Purging Eating Disorders Course and Outcome in a Population-Based Study of Adolescents. J Abnorm Child Psychol 2013;41:1083-1096.

41. Field AE, Sonneville KR, Micali N, Crosby RD, Swanson SA, Laird NM, et al. Prospective Association of Common Eating Disorders and Adverse Outcomes. Pediatrics 2012;130:e289-e295. 
42. Kessler RC, Berglund PA, Chiu WT, Deitz AC, Hudson JI, Shahly V, et al. The Prevalence and Correlates of Binge Eating Disorder in the World Health Organization World Mental Health Surveys. Biol Psychiatry 2013;73:904-914.

43. Crow SJ, Peterson CB, Levine AS, Thuras P, Mitchell JE. A survey of binge eating and obesity treatment practices among primary care providers. Int J Eat Disord 2004;35:348-353.

44. Baumann T, Schneider C, Vollmar M, Wolters M. Schulen auf einen Blick. Wiesbaden: Statistisches Bundesamt, 2012.

45. Kurth BM, Schaffrath Rosario A. Die Verbreitung von Übergewicht und Adipositas bei Kindern und Jugendlichen in Deutschland: Ergebnisse des bundesweiten Kinderund Jugendgesundheitssurveys (KiGGS). Bundesgesundheitsblatt Gesundheitsforschung Gesundheitsschutz 2007;50:736-743.

46. Kurth BM, Ellert U. Estimated and Measured BMI and Self-Perceived Body Image of Adolescents in Germany: Part 1. General Implications for Correcting Prevalence Estimations of Overweight and Obesity. Obesity Facts 2010;3:181-190.

47. Kleiser C, Schaffrath Rosario A, Mensink GBM, Prinz-Langenohl R, Kurth B-M. Potential determinants of obesity among children and adolescents in Germany: results from the cross-sectional KiGGS study. BMC Public Health 2009;46. doi:10.1186/1471-2458-9-46

48. Hilbert A, Hartmann AS, Czaja J, Schoebi D. Natural course of preadolescent loss of control eating. J Abnorm Psychol 2013;122:684-693. 
LOC EATING IN ADOLESCENTS

TABLE 1. Sociodemographic characteristics of loss of control (LOC) eating

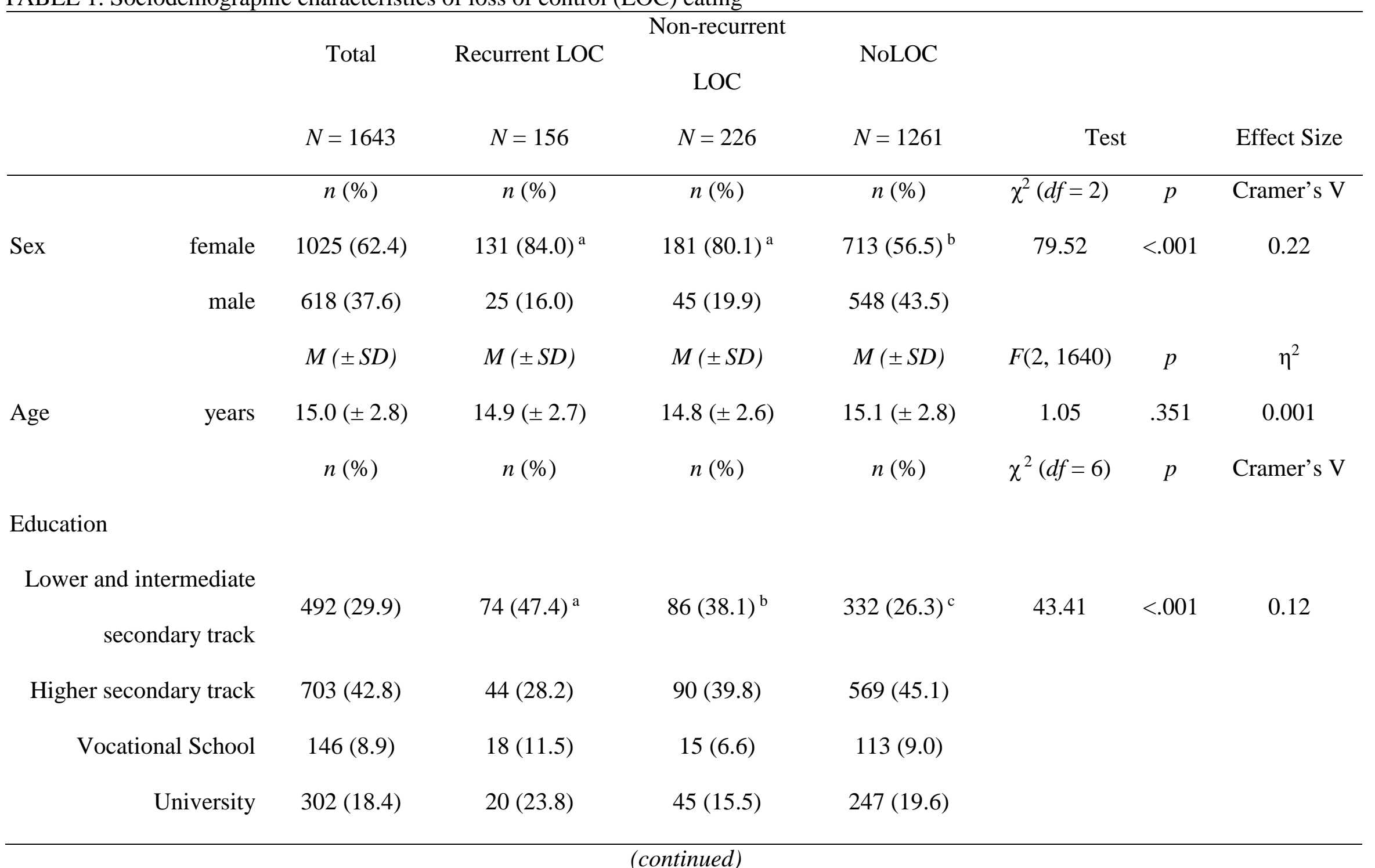


TABLE 1. Sociodemographic characteristics of loss of control (LOC) eating (continued)

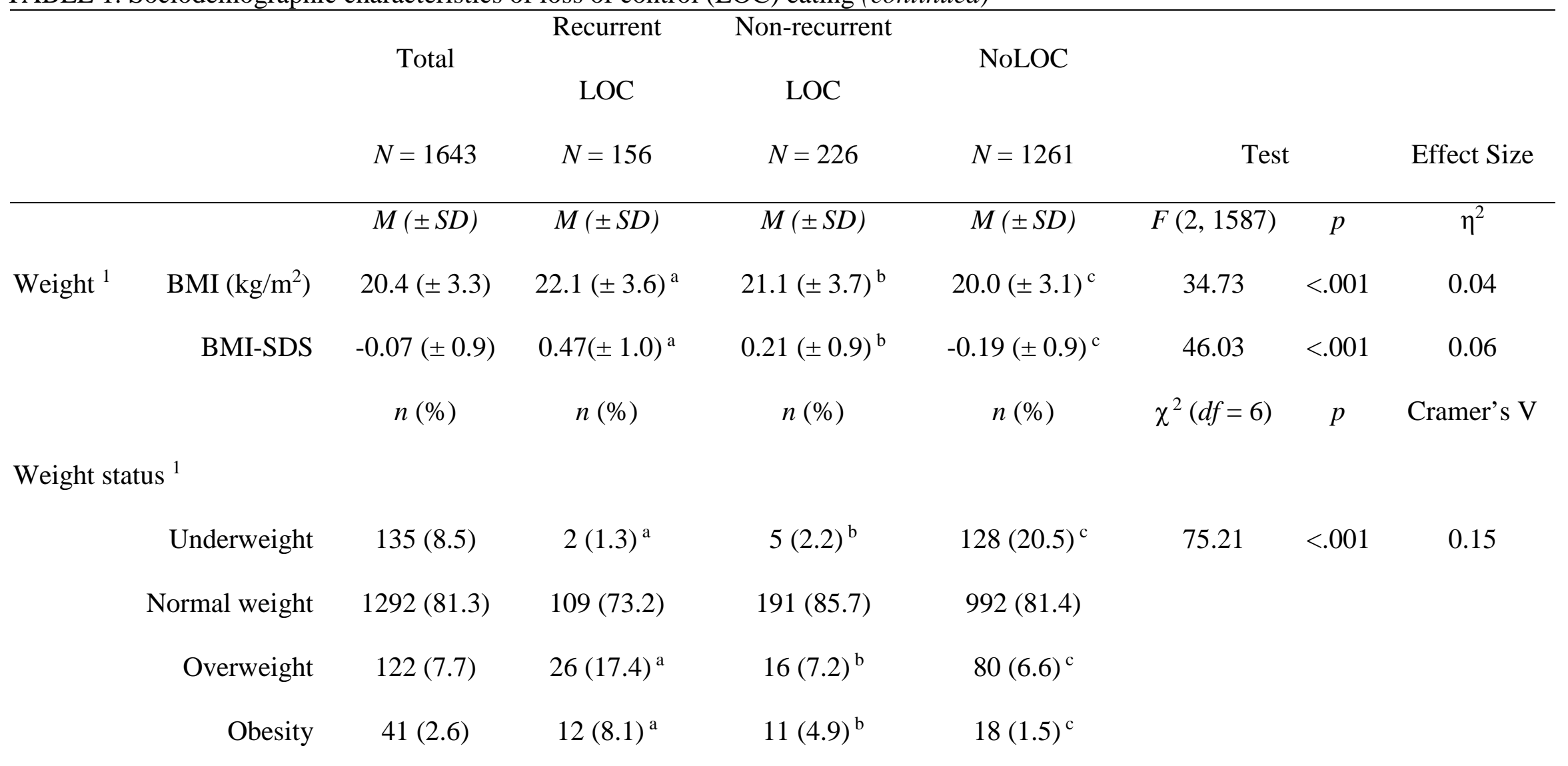

Note. NoLOC, No LOC eating episodes during the last 28 days; Non-recurrent LOC, < 4 LOC eating episodes during the last 28 days; Recurrent LOC, $\geq 4$ LOC eating episodes during the last 28 days; BMI, body mass index $\left(\mathrm{kg} / \mathrm{m}^{2}\right)$; BMI-SDS, body mass index-Standard Deviation Score; 


\section{LOC EATING IN ADOLESCENTS}

weight status based on German age- and sex-related reference data (Kromeyer-Hauschild et al., 2001): $<10^{\text {th }}$ percentile, underweight, $10-89^{\text {th }}$ percentile, normal weight, $90-97^{\text {th }}$ percentile, overweight, $>97^{\text {th }}$ percentile, obesity; ${ }^{1} n=1590$.

a, b, c Different superscripts indicate significant differences $(p<.05)$ 
TABLE 2. Clinical characteristics of loss of control (LOC) eating

\begin{tabular}{|c|c|c|c|c|c|c|c|}
\hline & Total & Recurrent & Non-recurrent & NoLOC & \multirow{3}{*}{\multicolumn{2}{|c|}{ Test }} & \multirow[b]{3}{*}{ Effect Size } \\
\hline & & LOC & LOC & & & & \\
\hline & $N=1643$ & $N=156$ & $N=226$ & $N=1261$ & & & \\
\hline EDE-Q8 $^{1}$ & $M( \pm S D)$ & $M( \pm S D)$ & $M( \pm S D)$ & $M( \pm S D)$ & $F(2,1640)$ & $p$ & $\eta^{2}$ \\
\hline \multirow[t]{2}{*}{ Global Score } & $1.4( \pm 1.5)$ & $3.5( \pm 1.4)^{\mathrm{a}}$ & $2.4( \pm 1.4)^{b}$ & $1.0( \pm 1.2)^{\mathrm{c}}$ & 240.69 & $<.001$ & 0.23 \\
\hline & $n(\%)$ & $n(\%)$ & $n(\%)$ & $n(\%)$ & $\chi^{2}(d f=2)$ & $p$ & Cramer's V \\
\hline$\geq 3.13$ & 288 (17.5) & $107(68.6)^{\mathrm{a}}$ & $78(34.5)^{b}$ & $103(8.2)^{\mathrm{c}}$ & 402.88 & $<.001$ & 0.50 \\
\hline$<3.13$ & 1355 (82.5) & 49 (31.4) & 148 (65.5) & 1158 (91.8) & & & \\
\hline CIA $^{1}$ & $M( \pm S D)$ & $M( \pm S D)$ & $M( \pm S D)$ & $M( \pm S D)$ & $F(2,1640)$ & $p$ & $\eta^{2}$ \\
\hline \multirow[t]{2}{*}{ Global Score } & $4.8( \pm 6.7)$ & $15.0( \pm 9.8)^{\mathrm{a}}$ & $7.9( \pm 6.9)^{b}$ & $3.0( \pm 4.4)^{\mathrm{c}}$ & 264.59 & $<.001$ & 0.25 \\
\hline & $n(\%)$ & $n(\%)$ & $n(\%)$ & $n(\%)$ & $\chi^{2}(d f=2)$ & $p$ & Cramer's V \\
\hline$\geq 16$ & 137 (8.3) & $64(41.0)^{\mathrm{a}}$ & $34(15.0)^{b}$ & $39(3.1)^{\mathrm{c}}$ & 276.77 & $<.001$ & 0.41 \\
\hline$<16$ & 1506 (91.7) & $92(59.0)$ & $192(85.0)$ & 1222 (96.9) & & & \\
\hline
\end{tabular}


LOC EATING IN ADOLESCENTS

TABLE 2. Clinical characteristics of loss of control (LOC) eating (continued)

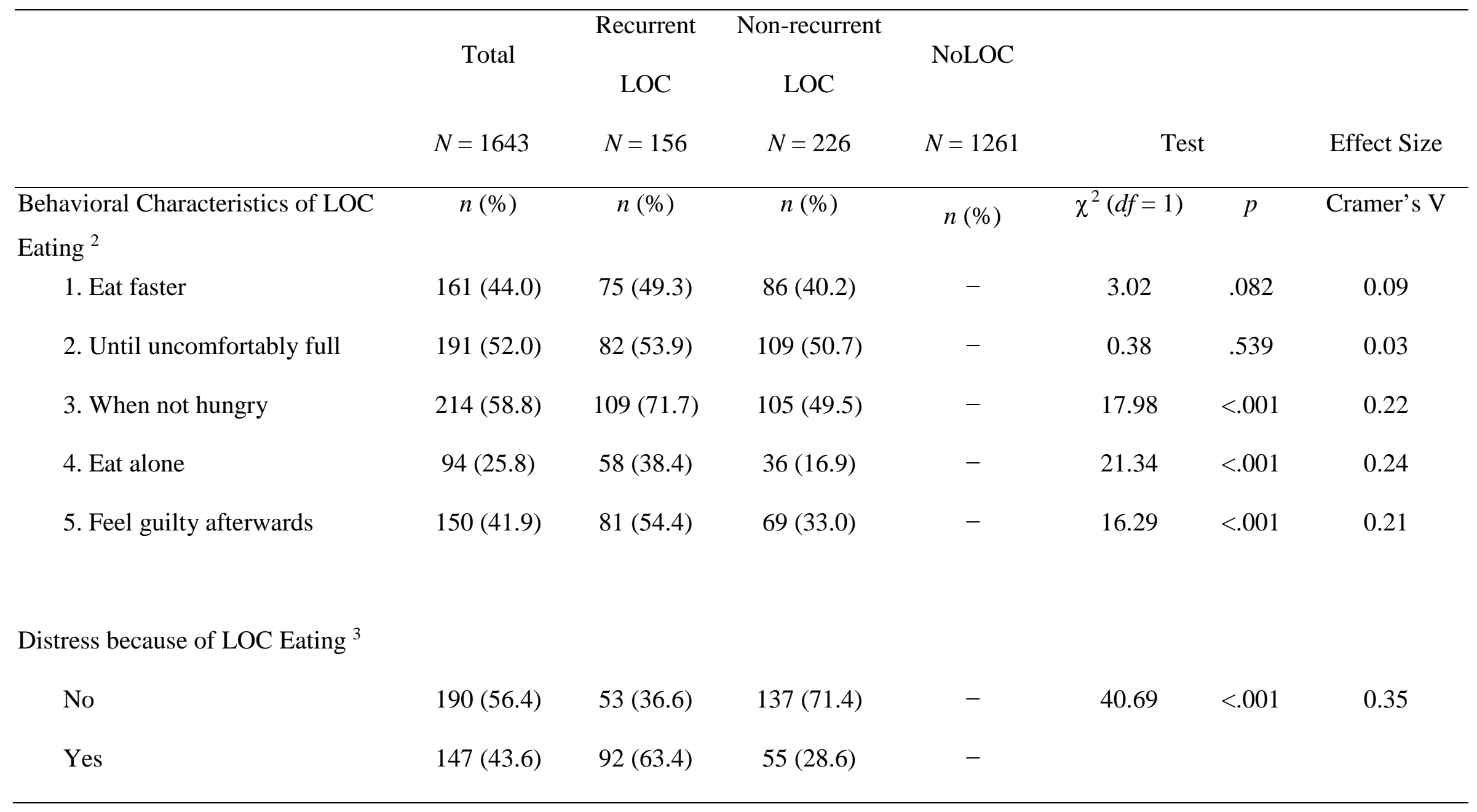




\section{LOC EATING IN ADOLESCENTS}

Note. Multivariate analysis of covariance, adjusted for sex, education, and weight status; NoLOC, No LOC eating episodes during the last 28 days; Non-recurrent LOC, < 4 LOC eating episodes during the last 28 days; Recurrent LOC, $\geq 4$ LOC eating episodes during the last 28 days; EDE-Q8,

Eating Disorder Examination-Questionnaire 8; CIA, Clinical Impairment Asssessment; ${ }^{1} n=1643 ;{ }^{2} n=358-367 ;{ }^{3} n=337$.

a, b, c Different superscripts indicate significant differences $(p<.05)$ 